\title{
Design Criteria of 2-DOF Planar Parallel Manipulator with Flexible Joints
}

\author{
F. A. Lara-Molina \\ E. H. Koroishi \\ Departement of Mechanical Engineering \\ Federal University of Technology - Paraná \\ Cornélio Procópio-PR, Brazil \\ Email: \{fabianmolina,edsonh\}@utfpr.edu.br
}

\author{
D. Dumur \\ Laboratoire des Signaux et Systèmes \\ CentraleSupélec-CNRS-Univ. Paris-Sud, Univ. Paris-Saclay \\ Control Department, 91192 \\ Gif sur Yvette cedex, France \\ Email: Didier.Dumur@centralesupelec.fr
}

\begin{abstract}
Design criteria of the parallel robots are required in order to perform the optimal design. This paper aims at proposing optimal design criteria for a planar parallel robot with flexible joints. Consequently, dynamic and elastodynamic criteria are examined with the purpose of analyzing their behavior as a function of the design variables that are the lengths of the links of the robot. The dynamic and elastodynamic design criteria are evaluated numerically.
\end{abstract}

\section{INTRODUCTION}

Kinematic design criteria of planar parallel robot have been extensively studied and well characterized [1], [2], [3], [4]. However, there are few contributions related to design criteria based on the dynamic performance of parallel robots with flexible links and joints. This issue is important for the mechanical design process and also for the further synthesis of the motion control algorithms [5].

Several contributions about parallel robots considering flexible links or joints have been addressed to formulate the elastodynamic model [6], motion control [5], [7] and the optimization of the mechanical structure [8], [9]. Nevertheless, this contribution aims at analyzing the behavior of design criteria based on the dynamic and elastodynamic performance of the robot as a function of the length of the links that are considered as the design variables.

In order to evaluate the design criteria, initially, the complete model of the 2-DOF planar parallel robot is formulated taking into account the flexible joints. In addition, the mechanism of the 2-DOF planar parallel robot has been considered with a symmetric mechanical structure in order to study its mechanical properties [1]. In addition, the symmetrical mechanism has been applied in parallel robot prototypes [10], [11]. Then, the design space is established. The dynamic and elastodynamic design criteria are further formulated. Finally, the design criteria are evaluated within the design space by means of numerical simulation.

\section{Roвot Modeling}

The 2-DOF planar parallel robot has two kinematic chains. Each kinematic chain includes an active or actuated joint, denoted as $A_{i}$, a passive or free joint, denoted as $B_{i}$ for $i=$ 1,2 , and two rigid links. The geometry of planar parallel robot is defined according to Fig. 1. The active joints are rotational and they are located at the Cartesian coordinates $\left(x_{a_{i}}, 0\right)^{T}$, for $i=1,2$. Additionally, the flexibility is considered in active joint, this flexibility is modeled as an elastic torsional spring $k_{i}$ which couples the rotors of the motor with the links. In this contribution the robot is considered as symmetric, thus the length of the links are defined by $\bar{r}_{1}, \bar{r}_{2}$. The end effector of the mechanism is located at $\mathbf{p}$ where its position is defined by the Cartesian coordinates $\left(\bar{x}_{p}, \bar{y}_{p}\right)$. Additionally, the fixed reference frame $O$ is defined in the middle of $A_{1} A_{2}$. The gravity acceleration acts perpendicular to the plane $x y$ in which the mechanism works.

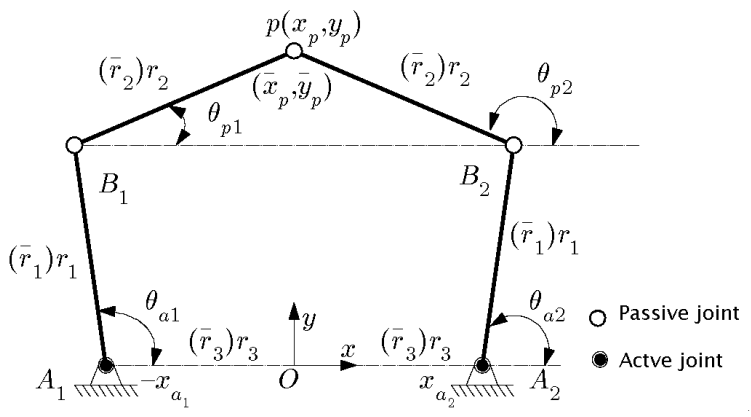

Fig. 1. The 2-DOF Planar Parallel Robot

For the symmetrical parallel mechanism, the link lengths are stated by $\bar{r}_{1}, \bar{r}_{2}$ and $\bar{r}_{3}$ (see Fig. 1). The link lengths are defined between zero and infinite. nevertheless, this dimensional length is eliminated in order to establish the design space as was presented in previous contributions [3]. In accordance with that, it is defined as $D=\left(\bar{r}_{1}+\bar{r}_{2}+\bar{r}_{3}\right) / 3$, thus, the three non-dimensional parameters $\left(r_{i}\right.$, for $\left.i=1,2,3\right)$ are defined by:

$$
r_{1}=\bar{r}_{1} / D \quad r_{2}=\bar{r}_{2} / D \quad r_{3}=\bar{r}_{3} / D
$$

Therefore:

$$
r_{1}+r_{2}+r_{3}=3
$$

Moreover, the end effector coordinates are also nondimensionalized as follows:

$$
x_{p}=\bar{x}_{p} / D \quad y_{p}=\bar{y}_{p} / D
$$




\section{A. Kinematic Model}

The position, $P$, of the end effector with respect to the fixed reference frame $O$ is defined by the Cartesian vector $\mathbf{p}=\left[\begin{array}{ll}x_{p} & y_{p}\end{array}\right]^{T}$. Additionally, the position of the points $B_{i}$ (for $i=1,2$ ) with respect to the fixed frame $O$ is defined by the vector $\mathbf{b}_{1}=\left[\begin{array}{ll}r_{1} \cos \left(\theta_{a 1}\right)-r_{3} & r_{1} \sin \left(\theta_{a 1}\right)\end{array}\right]^{T}$ and $\mathbf{b}_{2}=$ $\left[\begin{array}{ll}r_{1} \cos \left(\theta_{a 2}\right)+r_{3} & r_{1} \sin \left(\theta_{a 2}\right)\end{array}\right]^{T}$, respectively, with $\theta_{a 1}$ and $\theta_{a 2}$ being the active joint angles. The inverse kinematics is solved with the aids of the constraint equation $\left|\mathbf{b}_{i} \mathbf{p}\right|=r_{2}$, therefore:

$$
\begin{aligned}
& \left(x_{p}-r_{1} \cos \left(\theta_{a 1}\right)+r_{3}\right)^{2}+\left(y_{p}-r_{1} \sin \left(\theta_{a 1}\right)\right)^{2}=r_{2}^{2} \\
& \left(x_{p}-r_{1} \cos \left(\theta_{a 2}\right)-r_{3}\right)^{2}+\left(y_{p}-r_{1} \sin \left(\theta_{a 2}\right)\right)^{2}=r_{2}^{2}
\end{aligned}
$$

1) Jacobian Matrix: In order to derive the Jacobian matrix of the mechanism Eqs. (4) and (5) are differentiated with respect to time in order to obtain the following expression:

$$
\begin{aligned}
& r_{1}\left(y_{p} \cos \left(\theta_{a 1}\right)-\left(x_{p}+r_{3}\right) \sin \left(\theta_{a 1}\right)\right) \dot{\theta}_{a 1} \\
& =\left(x_{p}+r_{3}-r_{1} \cos \left(\theta_{a 1}\right)\right) \dot{x}_{p}+\left(y_{p}-r_{1} \sin \left(\theta_{a 1}\right)\right) \dot{y}_{p} \\
& r_{1}\left(y_{p} \cos \left(\theta_{a 2}\right)+\left(r_{3}-x_{p}\right) \sin \left(\theta_{a 2}\right)\right) \dot{\theta}_{a 2} \\
& =\left(x_{p}-r_{3}-r_{1} \cos \left(\theta_{a 2}\right)\right) \dot{x}_{p}+\left(y_{p}-r_{1} \sin \left(\theta_{a 2}\right)\right) \dot{y}_{p}
\end{aligned}
$$

the Eqs. (6) and (7) are written in the matrix form:

$$
\mathbf{A} \dot{\theta} a=\mathbf{B} \dot{\mathbf{p}}
$$

where $\dot{\mathbf{p}}=\left[\begin{array}{ll}\dot{x}_{p} & \dot{y}_{p}\end{array}\right]^{T}, \dot{\theta} a=\left[\begin{array}{ll}\dot{\theta}_{a 1} & \dot{\theta}_{a 2}\end{array}\right]^{T}$ and the $2 \times 2$ matrices $\mathbf{A}$ and $\mathbf{B}$ :

$$
\mathbf{A}=\left[\begin{array}{cc}
y_{p} c_{a 1}-\left(x_{p}+r_{3}\right) s_{a 1} & 0 \\
0 & y_{p} c_{a 2}+\left(r_{3}-x_{p}\right) s_{a 2}
\end{array}\right]
$$
and $\sin \left(\theta_{a i}\right)=s_{a i}$ for $i=1,2$.

The Jacobian matrix is expressed as:

$$
\mathbf{J}=\mathbf{A}^{-1} \mathbf{B}
$$

2) Workspace: In the design process, the Maximum Inscribed Circle (MIC) is an index useful to evaluate the flatness of the usable workspace, the MIC is inscribed within the usable workspace and it is tangent with singular loci [1]. The Maximum Inscribed Workspace (MIW) is defined as the workspace bounded by the MIC. The MIC is characterized by the expression:

$$
x^{2}+\left(y-y_{M I C}\right)^{2}=r_{M I C}^{2}
$$

where $r_{M I C}$ is the radius and $\left(0, y_{M I C}\right)$ is the center. For the cases when $r_{1}+r_{3}<r_{2}$, the MIC is defined by

$$
\begin{aligned}
r_{M I C} & =\left(r_{1}+r_{2}-\left|r_{1}-r_{2}\right|\right) / 2 \\
y_{M I C} & =\sqrt{\left(r_{1}+r_{2}+\left|r_{1}-r_{2}\right|\right)^{2} / 4-r_{3}^{2}}
\end{aligned}
$$

For the cases when $r_{1}+r_{3}>r_{2}$, the radius and center of the MIC are defined by:

$$
\begin{aligned}
& r_{M I C}=\left|y_{M I C}\right|-y_{c o l} \\
& y_{M I C}=\frac{\left(r_{1}+r_{2}+y_{c o l}\right)^{2}-r_{3}^{2}}{2\left(r_{1}+r_{2}+y_{c o l}\right)}
\end{aligned}
$$

with $y_{c o l}=\sqrt{r_{1}^{2}-\left(r_{2}-r_{3}\right)^{2}}$. Figure 2 shows the workspace for two different sets of non-dimensional lengths of the link of the mechanism. Fig. 2(a) presents the usable and MIW for $r_{1}=1.2, r_{2}=1$ and $r_{3}=0.8$ and Fig. 2(b) presents the usable workspace and MIW for $r_{1}=1.2, r_{2}=0.8$ and $r_{3}=1$. One can observe for these two cases that the MIW depends on the definition of the length of the links.

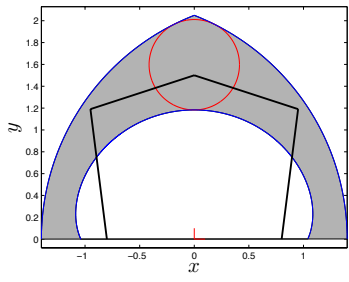

(a)

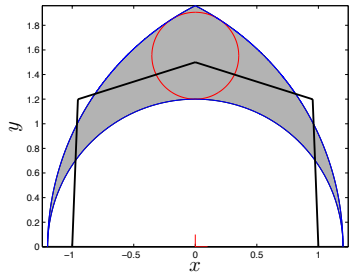

(b)
Fig. 2. Maximum Inscribed Workspace.

\section{B. Dynamic Model}

In order to derive the complete dynamic model of the robot, the dynamics of each kinematic chain together with the flexibility of the active joint is considered separately. Then, the kinematic constraints of the mechanism are applied to the dynamics of both kinematic chains to obtain the total dynamics of the robot.

Considering the dynamic parameters of the robot, the mass of links and stiffness of joints have to be defined as nondimensional parameters in order to evaluate the dynamic and elastodynamic performance. In accordance with that, let note that $m_{t}=\left(\bar{m}_{1 i}+\bar{m}_{2 i}\right) / 2$ and $k_{t}=\left(\bar{k}_{1}+\bar{k}_{2}\right) / 2$. Consequently, the non-dimensional masses of the links $\left(m_{1}\right.$ and $\left.m_{2}\right)$ and the stiffness of the joints are defined as:

$$
\begin{aligned}
& m_{1 i}=\bar{m}_{1 i} / m_{t} \quad m_{2 i}=\bar{m}_{2 i} / m_{t} \quad m_{1 i}+m_{2 i}=2 \\
& k_{1}=\bar{k}_{1 i} / k_{t} \quad k_{2}=\bar{k}_{2 i} / k_{t} \quad k_{1}+k_{2}=2
\end{aligned}
$$

The inertial moment and center of mass of the links are defined as function of the non-dimensional masses and lengths of the links as fallows:

$$
\begin{aligned}
d_{1 i} & =r_{1} / 2 & d_{2 i} & =r_{2} / 2 \\
I_{z 1 i} & =\frac{1}{12} m_{1} r_{1}^{2} & I_{z 2 i} & =\frac{1}{12} m_{2} r_{2}^{2}
\end{aligned}
$$

1) Dynamic of the Kinematic Chain: Figure 3(a) shows the model of a flexible joint. This flexibility is considered in the active joints. $\boldsymbol{\tau}_{m}=\left[\begin{array}{ll}\tau_{m 1} & \tau_{m 2}\end{array}\right]^{T}$ is the torque applied by the motors after the reduction, $\boldsymbol{\theta}_{m}=\left[\begin{array}{ll}\theta_{m 1} & \theta_{m 2}\end{array}\right]^{T}$ is the angular position of the motor after the reduction and $\boldsymbol{\theta}_{a}=$ $\left[\begin{array}{ll}\theta_{a 1} & \theta_{a 2}\end{array}\right]^{T}$ is the angular position of the active joints. The flexible transmission of the motor to the link of the parallel robot is modeled by a torsional spring of stiffness $k_{i}$ for $i=$ 1,2 . The inertia of the rotor of the motor after the reduction $\left(n_{i}\right)$ is defined by $j_{i}=I_{r i_{z z}} n_{i}^{2}$. The dynamics of each motor is obtained by applying the Euler equation as follows:

$$
j_{i} \ddot{\theta}_{m i}+v_{m i} \dot{\theta}_{m i}+k_{i}\left(\theta_{m i}-\theta_{a i}\right)=\tau_{m i}
$$


with $v_{m i}$ being the viscous friction in the motor.

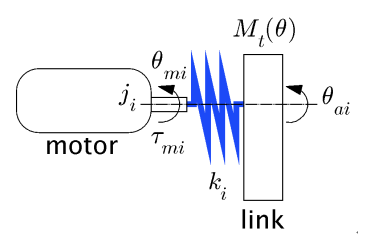

(a) Flexible joint.

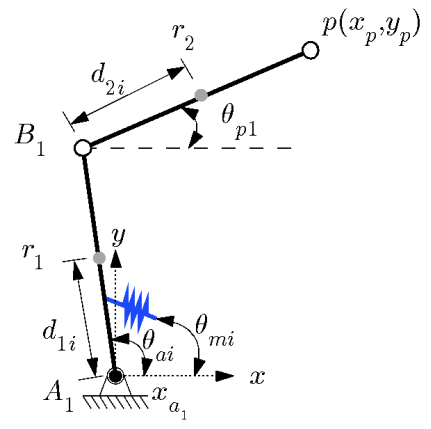

(b) Kinematic chain of the robot.
Fig. 3. Kinematic chain with flexibility in the active joint.

Figure 3(b) shows one of the kinematic chains. The modeling of each single kinematic chain is analyzed separately in order to simplify the total formulation of the dynamics of the robot. The dynamic equation is obtained by means of the Lagrange formulation presented in Eq. (14). This approach takes into account the kinetic and potential energies of the mechanism according to [12].

$$
\boldsymbol{\tau}_{i}-\mathbf{f}_{i}=\frac{d}{d t}\left(\frac{\partial L_{i}}{\partial \dot{\boldsymbol{\theta}}_{i}}\right)-\frac{\partial L_{i}}{\partial \boldsymbol{\theta}_{i}}
$$

where $\boldsymbol{\tau}_{i}=\left(\tau_{a i}, \tau_{p i}\right)^{T}$ is the vector of the joint torque, $\mathbf{f}_{i}=$ $\left(f_{a i}, f_{p i}\right)^{T}$ is the vector of the active and passive joint friction, $\boldsymbol{\theta}_{i}=\left(\theta_{a i}, \theta_{p i}\right)^{T}$ is the vector of joints for each $i-t h$ kinematic chain. Moreover, $L_{i}$ is the Lagrangian defined by:

$$
L_{i}=K_{i}-P_{i}
$$

with $K_{i}$ and $P_{i}$ being the kinematic and potential energy, respectively. As the gravity is acting in the $-z$ axis direction, the potential energy takes into account the elastic potential energy of the active joint, thus

$$
P_{i}=\frac{1}{2} k_{i}\left(\theta_{a i}-\theta_{m i}\right)^{2}
$$

The total kinetic energy is $K_{i}=K_{1 i}+K_{2 i}$, where $K_{1 i}$ and $K_{2 i}$ represent the kinetic energy of the first and second link of the $i-t h$ kinematic chain respectively (see Fig. 3(b)), thus:

$$
\begin{aligned}
K_{1 i} & =\frac{1}{2} I_{z 1 i} \dot{\theta}_{a i}^{2}+\frac{1}{2} m_{1 i}\left(\dot{x}_{c 1 i}^{2}+\dot{y}_{c 1 i}^{2}\right) \\
K_{2 i} & =\frac{1}{2} I_{z 2 i} \dot{\theta}_{p i}^{2}+\frac{1}{2} m_{2 i}\left(\dot{x}_{c 2 i}^{2}+\dot{y}_{c 2 i}^{2}\right)
\end{aligned}
$$

$m_{1 i}$ and $m_{2 i}$ are the masses, and $I_{z 1 i}$ and $I_{z 2 i}$ are the inertia moments of the links for each $i-t h$ kinematic chain. Additionally, $\left(x_{c 1 i}, y_{c 1 i}\right)^{T}$ and $\left(x_{c 2 i}, y_{c 2 i}\right)^{T}$ are the Cartesian positions of the center of mass of each link that are defined by:

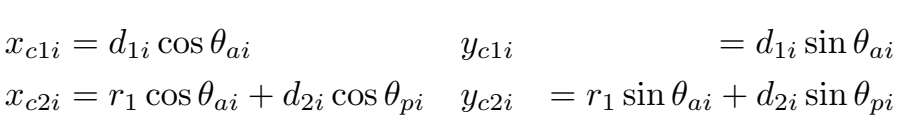

By substituting the previous relations in the Lagrangian of Eq. (15), it is obtained:

$$
\begin{aligned}
L_{i} & =\frac{1}{2} \dot{\theta}_{a i}^{2} \alpha_{i}+\frac{1}{2} \dot{\theta}_{p i}^{2} \beta_{i}+\gamma_{i} \dot{\theta}_{a i} \dot{\theta}_{p i} \cos \left(\theta_{a i}-\theta_{p i}\right)-\ldots \\
& \ldots \frac{1}{2} k_{i}\left(\theta_{a i}-\theta_{m i}\right)^{2}
\end{aligned}
$$

where $\alpha_{i}=I_{z 1 i}+m_{1 i} d_{1 i}^{2}+m_{2 i} r_{1}^{2}, \beta_{i}=I_{z 2 i}+m_{2 i} d_{2 i}^{2}$ and $\gamma_{i}=m_{2 i} r_{1} d_{2 i}$.

The dynamics of each $i-t h$ kinematic chain is obtained by substituting the Lagrangian of Eq. (19) into the Lagrange formulation presented in Eq. (14), thus:

$$
\boldsymbol{\tau}_{i}-\mathbf{f}_{i}=\mathbf{M}_{i}\left(\boldsymbol{\theta}_{i}\right) \ddot{\boldsymbol{\theta}}_{i}+\mathbf{C}_{i}\left(\boldsymbol{\theta}_{i}, \dot{\boldsymbol{\theta}}_{i}\right) \dot{\boldsymbol{\theta}}_{i}+\mathbf{f}_{k i}
$$

where

$$
\begin{aligned}
& \mathbf{M}_{i}\left(\boldsymbol{\theta}_{i}\right)=\left[\begin{array}{cc}
\alpha_{i} & \gamma_{i} \cos \left(\theta_{a i}-\theta_{p i}\right) \\
\gamma_{i} \cos \left(\theta_{a i}-\theta_{p i}\right) & \beta_{i}
\end{array}\right] \\
& \mathbf{C}_{i}\left(\boldsymbol{\theta}_{i}, \dot{\boldsymbol{\theta}}_{i}\right)=\left[\begin{array}{cc}
0 & \gamma_{i} \dot{\theta}_{p i} \sin \left(\theta_{a i}-\theta_{p i}\right) \\
-\gamma_{i} \dot{\theta}_{a i} \sin \left(\theta_{a i}-\theta_{p i}\right) & 0
\end{array}\right] \\
& \mathbf{f}_{k i}=\left[k_{i}\left(\theta_{a i}-\theta_{m i}\right) \quad 0\right]^{T}
\end{aligned}
$$

2) Dynamic Model: Finally, the complete dynamic model of the two kinematic chains is formulated by combining the model of the two kinematic chains of Eq. (20), thus:

$$
\mathbf{M}(\boldsymbol{\theta}) \ddot{\boldsymbol{\theta}}+\mathbf{C}(\boldsymbol{\theta}, \dot{\boldsymbol{\theta}}) \dot{\boldsymbol{\theta}}+\mathbf{f}+\mathbf{f}_{k}=\boldsymbol{\tau}
$$

with $\boldsymbol{\theta}=\left(\boldsymbol{\theta}_{a}^{T}, \boldsymbol{\theta}_{p}^{T}\right)^{T}, \dot{\boldsymbol{\theta}}=\left(\dot{\boldsymbol{\theta}}_{a}^{T}, \dot{\boldsymbol{\theta}}_{p}^{T}\right)^{T}, \mathbf{f}=\left(\mathbf{f}_{a}^{T}, \mathbf{f}_{p}^{T}\right)^{T}$ and $\boldsymbol{\tau}=\left(\boldsymbol{\tau}_{a}^{T}, \boldsymbol{\tau}_{p}^{T}\right)^{T} . \boldsymbol{\theta}_{p}=\left(\theta_{p 1}, \theta_{p 2}\right)^{T}$ is the vector of angular position of active joints. $\boldsymbol{\tau}_{a}=\left(\tau_{a 1}, \tau_{a 2}\right)^{T}$ is the vector of input torque in the active joints, $\boldsymbol{\tau}_{p}=\left(\tau_{p 1}, \tau_{p 2}\right)^{T}$ is the vector of the torque in the passive joints. $\mathbf{f}_{a}=\left(f_{a 1}, f_{a 2}\right)^{T}$ is the vector of frictions in the active joints, $\mathbf{f}_{p}=\left(f_{p 1}, f_{p 2}\right)^{T}$ is the vector of frictions in the passive joints. $\mathbf{f}_{k}=\left(\mathbf{f}_{k a}^{T}, \mathbf{f}_{k p}^{T}\right)^{T}$ is the elastic torque in active and passive joints respectively. The elastic torque is introduced in active joints by the elastic transmission of the motor, therefore $\mathbf{f}_{k a}=\left(k_{1}\left(\theta_{a 1}-\theta_{m 1}\right), k_{2}\left(\theta_{a 2}-\theta_{m 2}\right)\right)^{T}$. In addition, no elasticity is considered in passive points, thus $\mathbf{f}_{k p}=(0,0)^{T}$. An input torque is not applied in passive joints, thus $\boldsymbol{\tau}_{p}=(0,0)^{T}$. Moreover, the friction in passive joints is assumed negligible when compared with friction in active joints thus $\mathbf{f}_{p}=(0,0)^{T}$. The friction in the active joints considers the viscous friction, $v_{i} \dot{\theta}_{a i}$, and coulomb friction, $d_{i} \operatorname{sign}\left(\dot{\theta}_{a i}\right)$, thus:

$$
f_{a i}=v_{i} \dot{\theta}_{a i}+d_{i} \operatorname{sign}\left(\dot{\theta}_{a i}\right)
$$

The mass and Coriolis matrices (see Eq. (21)) of both kinematic chains are given by:

$\mathbf{M}(\boldsymbol{\theta})=\left[\begin{array}{cccc}\alpha_{1} & 0 & \gamma_{1} c_{a p 1} & 0 \\ 0 & \alpha_{2} & 0 & \gamma_{2} c_{a p 2} \\ \gamma_{1} c_{a p 1} & 0 & \beta_{1} & 0 \\ 0 & \gamma_{2} c_{a p 2} & 0 & \beta_{2}\end{array}\right]$
$\mathbf{C}(\boldsymbol{\theta}, \dot{\boldsymbol{\theta}})=\left[\begin{array}{cccc}0 & 0 & \gamma_{1} \dot{\theta}_{p 1} s_{a p 1} & 0 \\ 0 & 0 & 0 & \gamma_{2} \dot{\theta}_{p 2} s_{a p 2} \\ -\gamma_{1} \dot{\theta}_{a 1} s_{a p 1} & 0 & 0 & 0 \\ 0 & -\gamma_{2} \dot{\theta}_{a 2} s_{a p 2} & 0 & 0\end{array}\right]$

where $c_{a p i}=\cos \left(\theta_{a i}-\theta_{p i}\right), s_{a p i}=\sin \left(\theta_{a i}-\theta_{p i}\right)$, for $i=1,2$. 
3) Complete dynamic model: The dynamics of the two motors is written in matrix form by combining the model of the two single motors, of eq. (13), as follows:

$$
\mathbf{J} \ddot{\boldsymbol{\theta}}_{m}+\mathbf{V} \dot{\boldsymbol{\theta}}_{m}+\mathbf{K}\left(\boldsymbol{\theta}_{m}-\boldsymbol{\theta}_{a}\right)=\boldsymbol{\tau}_{m}
$$

with $\mathbf{J}=\operatorname{diag}\left(j_{1}, j_{2}\right)$ being the inertia matrix of rotors of motors, $\mathbf{K}=\operatorname{diag}\left(k_{1}, k_{2}\right)$ being the stiffness matrix of flexible transmission and $\mathbf{V}=\operatorname{diag}\left(v_{m 1}, v_{m 2}\right)$.

The complete dynamic model of the parallel mechanism is obtained by considering the coupling of the kinematic chains at the passive joint of point $P$. The kinematic constraints of the coupling are derived from the Jacobian matrix. By using the D'Alembert's principle and the principle of virtual work, the torques of the active joint $\tau_{a}$ and torque of the joints $\tau$ satisfy the relation:

$$
\tau_{a}=\Psi^{T} \boldsymbol{\tau}
$$

where $\Psi=\partial \boldsymbol{\theta} / \partial \boldsymbol{\theta}_{a}$, consequently $\Psi=[\mathbf{I}, \mathbf{J}]$ with $\mathbf{I}$ being the identity matrix and $\mathbf{J}=\partial \boldsymbol{\theta}_{p} / \partial \boldsymbol{\theta}_{a}$ where:

$$
\mathbf{J}=\frac{\partial \boldsymbol{\theta}_{p}}{\partial \boldsymbol{\theta}_{a}}=-\left[\frac{\partial \mathbf{h}}{\partial \boldsymbol{\theta}_{p}}\right]^{-1}\left[\frac{\partial \mathbf{h}}{\partial \boldsymbol{\theta}_{a}}\right]
$$

with:

$$
\mathbf{h}=\left[\begin{array}{c}
r_{1} c_{a 1}+r_{2} c_{p 1}-2 r_{3}-r_{1} c_{a 2}-r_{2} c_{p 2} \\
r_{1} s_{a 1}+r_{2} s_{p 1}-r_{1} s_{a 2}-r_{2} s_{p 2}
\end{array}\right]
$$

where $c_{p i}=\cos \theta_{p i}, s_{p i}=\sin \theta_{p i}$ for $i=1,2$. The dynamic equation of Eq. (21) is multiplied by the constraint of Eq. (24) leading to:

$$
\Psi^{T} \mathbf{M}(\boldsymbol{\theta}) \ddot{\boldsymbol{\theta}}+\Psi^{T} \mathbf{C}(\boldsymbol{\theta}, \dot{\boldsymbol{\theta}}) \dot{\boldsymbol{\theta}}+\Psi^{T} \mathbf{f}+\Psi^{T} \mathbf{f}_{k}=\Psi^{T} \boldsymbol{\tau}
$$

Moreover, the following relations are established:

$$
\begin{aligned}
\dot{\boldsymbol{\theta}} & =\frac{\partial \boldsymbol{\theta}}{\partial \boldsymbol{\theta}_{a}} \dot{\boldsymbol{\theta}}_{a} \\
\ddot{\boldsymbol{\theta}} & =\dot{\Psi} \dot{\boldsymbol{\theta}}_{a}+\Psi \ddot{\boldsymbol{\theta}}_{a}
\end{aligned}
$$

The relation of Eqs. (24), (27) and (28) are substituted into Eq. (26), therefore the total dynamic equation is expressed as follows:

$$
\mathbf{M}_{t} \ddot{\boldsymbol{\theta}}_{a}+\mathbf{C}_{t} \dot{\boldsymbol{\theta}}_{a}+\mathbf{f}_{a}+\mathbf{f}_{k a}=\boldsymbol{\tau}_{a}
$$

where $\mathbf{M}_{t}=\Psi^{T} \mathbf{M}(\boldsymbol{\theta}) \Psi$ and $\mathbf{C}_{t}=\Psi^{T} \mathbf{M}(\boldsymbol{\theta}) \dot{\Psi}+$ $\Psi^{T} \mathbf{C}(\boldsymbol{\theta}, \dot{\boldsymbol{\theta}}) \Psi$.

Moreover, the complete dynamic model considers the dynamics of the motor defined in eq. (23) and the dynamics of the parallel mechanism of eq. (29), thus:

$$
\begin{cases}\mathbf{M}_{t} \ddot{\boldsymbol{\theta}}_{a}+\mathbf{C}_{t} \dot{\boldsymbol{\theta}}_{a}+\mathbf{f}_{a}+\mathbf{K}\left(\boldsymbol{\theta}_{a}-\boldsymbol{\theta}_{m}\right) & =0 \\ \mathbf{J} \ddot{\boldsymbol{\theta}}_{m}+\mathbf{V} \dot{\boldsymbol{\theta}}_{m}+\mathbf{K}\left(\boldsymbol{\theta}_{m}-\boldsymbol{\theta}_{a}\right) & =\boldsymbol{\tau}_{m}\end{cases}
$$

The torque is only applied by the motors and no torque is applied directly to active joints, thus $\tau_{a}=(0,0)^{T}$ in eq. (30). One can observe that parallel mechanism and motor dynamics of eq. (30) are dynamically coupled by the elastic torque in the active joints $\mathbf{K}\left(\boldsymbol{\theta}_{m}-\boldsymbol{\theta}_{a}\right)$. Finally, the dynamics can be express by using the matrix notation as follows:

$$
\mathbf{M}_{T}(\boldsymbol{\theta}) \ddot{\mathbf{z}}+\mathbf{C}_{T}(\boldsymbol{\theta}, \dot{\boldsymbol{\theta}}) \dot{\mathbf{z}}+\mathbf{f}_{t}+\mathbf{K}_{T} \mathbf{z}=\mathbf{u}
$$

where $\mathbf{z}=\left(\boldsymbol{\theta}_{a}^{T}, \boldsymbol{\theta}_{m}^{T}\right)^{T}, \quad \mathbf{M}_{T}(\boldsymbol{\theta})=\left[\begin{array}{cc}\mathbf{M}_{t}(\boldsymbol{\theta}) & 0_{2,2} \\ 0_{2,2} & \mathbf{J}\end{array}\right]$, $\mathbf{C}_{T}(\boldsymbol{\theta}, \dot{\boldsymbol{\theta}})=\left[\begin{array}{cc}\mathbf{C}_{t}(\boldsymbol{\theta}, \dot{\boldsymbol{\theta}}) & 0_{2,2} \\ 0_{2,2} & 0_{2,2}\end{array}\right]$ and $\mathbf{K}_{T}=$ $\left[\begin{array}{cccc}k_{1} & 0 & -k_{1} & 0 \\ 0 & k_{2} & 0 & -k_{2} \\ -k_{1} & 0 & k_{1} & 0 \\ 0 & -k_{2} & 0 & k_{2}\end{array}\right], \quad \mathbf{f}_{t}=\left(\mathbf{f}_{a}^{T}, \mathbf{V} \dot{\boldsymbol{\theta}}_{m}^{T}\right)^{T}$ and
$\mathbf{u}=\left(0_{1,2}, \boldsymbol{\tau}_{m}\right)^{T}$.

\section{PERFORMANCE INDICES}

Several performance criteria have been formulated in order to optimize the kinematic design of parallel mechanism [13]. The atlases of the performance indices i.e., the performance indices for every single length of the non-dimensional links can be evaluated based on the design space. This section first presentes the design space and then introduces the dynamic and elastodynamic performance criteria.

\section{A. The Design Space}

The optimum design demands the evaluation of a performance index as function of the link lengths in order to determine the link dimensions that optimize the performance of the mechanism. The design space assesses all the possible combinations of the links dimensions and their correspondent performance indices [2].

The non-dimensional length of links were previously described by eqs. (1) and (2). Theoretically, $0<r_{i}<3$, nevertheless, the non-dimensional lengths of the links are constrained in order to avoid a failure of mechanism assembly [2], thus:

$$
0<r_{1}, r_{2}<3 \quad \text { and } \quad 0 \leq r_{3} \leq 1.5
$$

Based on the Eq. (2) and the constraints of Eq. (32), the design space is defined as a trapezoid shown in Fig. 4(a). Additionally, a planar configuration (see Fig. 4) is defined based on two orthogonal coordinates $s$ and $t$, with

$$
\begin{aligned}
s & =2 r_{1} / \sqrt{3}+r_{3} / \sqrt{3} \\
t & =r_{3}
\end{aligned}
$$

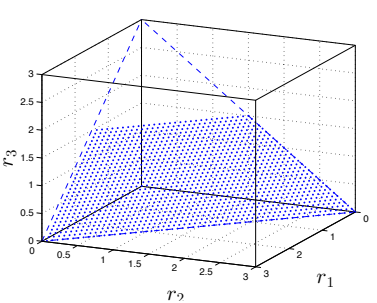

(a)

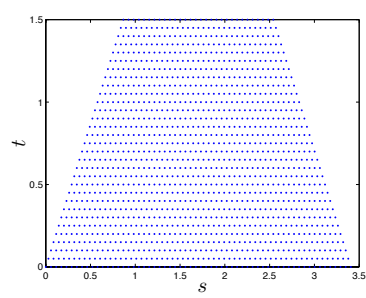

(b)
Fig. 4. Design space of the parallel symmetrical robot. 


\section{B. Dynamic Performance Evaluation}

The dynamic performance evaluates the dynamic dexterity, i.e. the capability of the end-effector to accelerate on every one of the directions of the workspace. The dynamic performance is evaluated by using the Generalized-Inertia-Ellipsoid (GIE) proposed by [14] that permits to quantify the inertia properties of the mechanism. With this purpose the inertia matrix $\mathbf{M}_{t}$ of links, previously defined in eq. (29), is used to evaluate the dynamic dexterity based on the condition number of inertia matrix in the workspace $\left(\mathbf{M}_{w}(\theta)=\mathbf{J}^{-T} \mathbf{M}_{t}(\boldsymbol{\theta}) J^{-1}\right)$ that measures isotropy of the acceleration ratio along the axes, thus:

$$
1 / k\left(\mathbf{M}_{w}(\boldsymbol{\theta})\right)=\frac{\sigma_{\min }\left(\mathbf{M}_{w}(\boldsymbol{\theta})\right)}{\sigma_{\max }\left(\mathbf{M}_{w}(\boldsymbol{\theta})\right)}
$$

where $\sigma_{\max }\left(\mathbf{M}_{w}(\boldsymbol{\theta})\right)$ and $\sigma_{\min }\left(\mathbf{M}_{w}(\boldsymbol{\theta})\right)$ are the maximum and minimum singular values of the workspace inertia matrix. One can observe that this measure depends on the posture of the robot, i.e., the dynamic dexterity is a local performance index and the desirable isotropic performance is obtained when $1 / k\left(\mathbf{M}_{w}(\boldsymbol{\theta})\right)=1$. The global dynamic dexterity considers the dexterity within the maximum inscribed workspace by using the following expression:

$$
\frac{\int_{M I W} 1 / k\left(\mathbf{M}_{w}(\boldsymbol{\theta})\right) d w}{\int_{M I W} d w}
$$

Maximizing the global dexterity leads to the optimal dynamic performance.

\section{Elastodynamic Performance Evaluation}

The elastodynamic performance evaluates at a specific posture of robot the natural modes and frequencies of the structure of the robot that are originated by flexible elements of the structure [15]. For this contribution, the active joints of the parallel robot are considered with flexibility. In order to evaluate the elastodynamic performance, the complete dynamics of the robot, previously defined in eq. (31), is assumed at a fixed posture and undamped, i.e., $\dot{\mathbf{z}}=(0,0,0,0)^{T}$ and $\mathbf{f}_{t}=(0,0,0,0)^{T}$, therefore these assumptions lead to:

$$
\mathbf{M}_{T}(\boldsymbol{\theta}) \ddot{\mathbf{z}}+\mathbf{K}_{T} \mathbf{z}=\mathbf{u}
$$

The elastodynamic performance depends on the posture of the robot because total inertia matrix of the robot $\left(\mathbf{M}_{T}(\boldsymbol{\theta})\right)$ is also posture dependent. Natural modes and frequencies are obtained by solving the associated eigenvalue problem when the dynamics of eq. (37) is evaluated for motions produced by non-equilibrium initial conditions and non-excitation i.e., $\mathbf{u}=(0,0,0,0)^{T}$, thus $\left(\mathbf{K}_{T}-\lambda_{T}^{2} \mathbf{M}_{T}(\boldsymbol{\theta})\right) \mathbf{z}=0$. Note $\lambda_{T}=$ $\left(\lambda_{1}, \ldots, \lambda_{n}\right)$ and $\phi_{T}=\left(\phi_{1}, \ldots, \phi_{n}\right)$ the set of eigenvalues and eigenvector respectively. It is desirable that the robot operates below the smallest mode in order to avoid undesirable vibrations during the motion. Therefore, the elastodynamic performance is evaluated by computing the smallest eigenvalue over the MIW, thus

$$
\lambda_{e}=\min _{M I W}\left(\lambda_{T}\left(r_{1}, r_{2}, r_{3}\right)\right)
$$

The elastodynamic performance is optimized by maximizing the smallest eigenvalue as function of the non-dimensional length of the links.

\section{RESUlTS}

The non-dimensional parameters adopted in this contribution are: $m_{1 i}=1.2, m_{2 i}=0.8$, this implied that the mass of the first link is 20 percent greater than the mass of the second link. In addition, $k_{1 i}=1$ and $k_{2 i}=1$, this means that the stiffness of active joints is equal. Additionally, a relationship between the non-dimensioal mass of the first link and the mass of the rotor was established based on parameters of previous contributions [16] as follows: $j_{i}=0.5 m_{1}$.

\section{A. Dynamic Performance}

Initially, the dynamic dexterity is evaluated at each single posture by considering the specific set of non-dimensional lengths of the links $r_{1}=1.2, r_{2}=1.0$ and $r_{3}=0.8$. Figure 5 shows the local dynamic dexterity $1 / k\left(\mathbf{M}_{w}(\boldsymbol{\theta})\right)$ for each single posture within the usable workspace. It is observed that the maximum and minimum values of local dynamic dexterity $\left(\max \left(1 / k\left(\mathbf{M}_{w}(\boldsymbol{\theta})\right)\right)\right.$ and $\left.\min \left(1 / k\left(\mathbf{M}_{w}(\boldsymbol{\theta})\right)\right)\right)$ are located within the MIW. i.e., the maximum acceleration ratio of the end-effector is obtained closed to the center of the maximum inscribed circle.

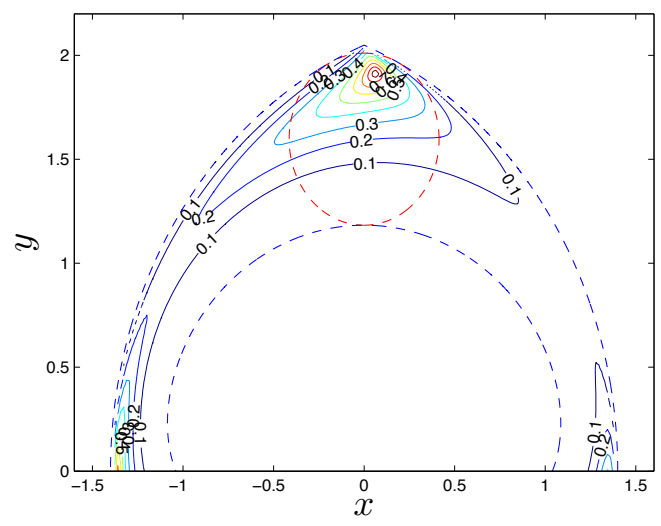

Fig. 5. Local dynamic dexterity over the usable workspace and MIW.

As an additional result, the global dynamic dexterity is evaluated over the design space by using the expression of eq. (36). Figure 6 shows the design space and the atlas of the global dynamic dexterity. It is observed a discontinuity of global dynamic dexterity for $r_{2}=r_{1}+r_{3}$, this discontinuity follows the same trend presented by kinematic performance criteria presented in previous contributions that depend on the Jacobian matrix [1]. The atlas of Fig. 6 indicates that with the decrease of $r_{3}$ the maximum global dynamic dexterity increases, since $t=r_{3}$.

\section{B. Elastodynamic Performance}

Figure 7 shows the atlas of the elastodynamic performance that was computed by using the expression of eq. (38). The 


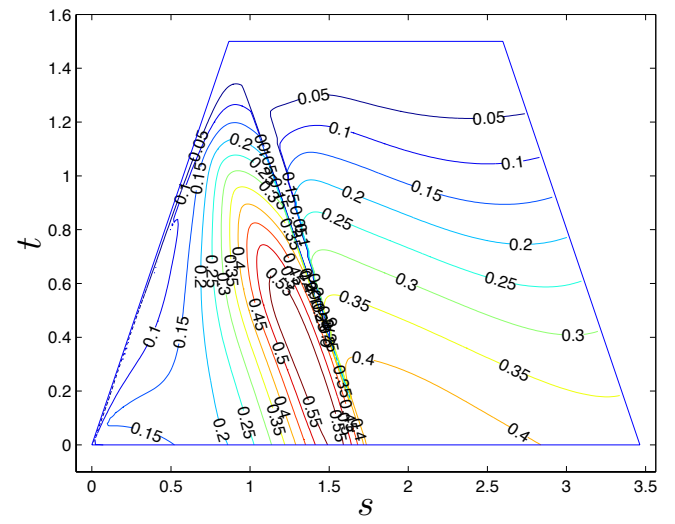

Fig. 6. Atlas of global dynamic dexterity.

atlas indicates that the elastodynamic performance depends principally on $s$. Moreover, it is observed in the atlas of Fig. 7 that elastodynamic performance shows a rather small dependence on the on $t=r_{3}$. Consequently, the elastodynamic performance depends on the $r_{1}$ and $r_{2}$ since the length of this link is directly proportional to the total inertia matrix of the robot $\mathbf{M}_{T}$ defined in eq. (37). Hence, based on the definition of eqs. (33) and (34), the smallest eigenvalue maximized by selecting links lengths that follow the relation $r_{1} \leq r_{2}$, this region in located in the left border of the design space showed in Fig. 7.

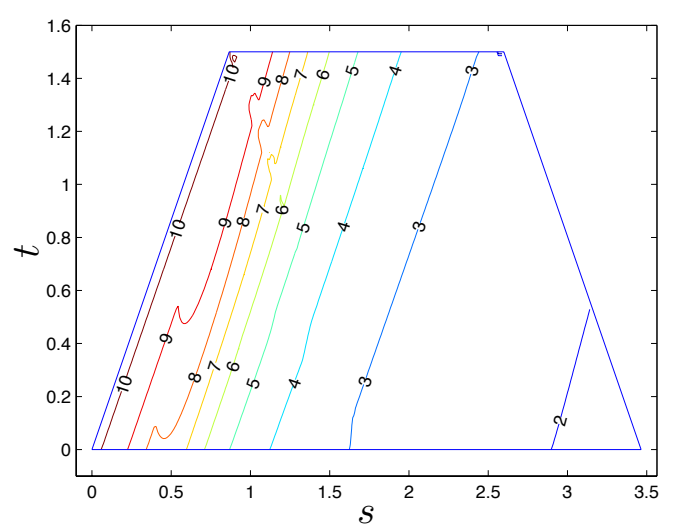

Fig. 7. Atlas of elastodynamic performance.

\section{CONCLUSION}

This paper presented design criteria based on dynamic and elastodynamic model of planar parallel mechanisms. The dynamic performance was evaluated by using the global dynamic dexterity and the elastodynamic performance was evaluated based on the solution of eigenvalue problem. The global dynamic dexterity and eigenvalues of the structure were evaluated within the design space, consequently, the atlases of these design criteria were established.
This contribution is an initial tentative toward the optimal design of the parallel robot with flexible joints. Future works will encompass the optimal design of parallel robots with flexible joints by taking into account the design criteria presented in this contribution.

\section{ACKNOWLEDGMENT}

The authors express their acknowledgements to the Graduate Program in Mechanical Engineering of the Federal University of Technology - Paraná funded by CAPES.

\section{REFERENCES}

[1] X.-J. Liu, J. Wang, and G. Pritschow, "Performance atlases and optimum design of planar 5r symmetrical parallel mechanisms," Mechanism and machine theory, vol. 41, no. 2, pp. 119-144, 2006.

[2] X.-J. Liu, J. Wang, and G. Pritschow, "Kinematics, singularity and workspace of planar 5r symmetrical parallel mechanisms," Mechanism and Machine Theory, vol. 41, no. 2, pp. 145-169, 2006.

[3] F. Gao, X.-J. Liu, and X. Chen, "The relationships between the shapes of the workspaces and the link lengths of 3-dof symmetrical planar parallel manipulators," Mechanism and Machine Theory, vol. 36, no. 2, pp. 205-220, 2001.

[4] F. Lara-Molina, J. Rosário, and D. Dumur, "Multi-objective optimization of stewart-gough manipulator using global indices," in Advanced Intelligent Mechatronics (AIM), 2011 IEEE/ASME International Conference on, pp. 79-85, IEEE, 2011.

[5] X. Zhang, J. K. Mills, and W. L. Cleghorn, "Vibration control of elastodynamic response of a 3-prr flexible parallel manipulator using pzt transducers," Robotica, vol. 26, no. 05, pp. 655-665, 2008.

[6] M. Rognant, E. Courteille, and P. Maurine, "A systematic procedure for the elastodynamic modeling and identification of robot manipulators," Robotics, IEEE Transactions on, vol. 26, no. 6, pp. 1085-1093, 2010.

[7] X. Wang and J. K. Mills, "Fem dynamic model for active vibration control of flexible linkages and its application to a planar parallel manipulator," Applied Acoustics, vol. 66, no. 10, pp. 1151-1161, 2005.

[8] C. Alessandro and S. Rosario, "Elastodynamic optimization of a 3t1r parallel manipulator," Mechanism and Machine Theory, vol. 73, pp. 184196, 2014.

[9] H. Li, Z. Yang, and T. Huang, "Dynamics and elasto-dynamics optimization of a 2-dof planar parallel pick-and-place robot with flexible links," Structural and Multidisciplinary Optimization, vol. 38, no. 2, pp. 195-204, 2009.

[10] Y.-X. Zhang, S. Cong, W.-W. Shang, Z.-X. Li, and S.-L. Jiang, "Modeling, identification and control of a redundant planar 2-dof parallel manipulator," International Journal of Control Automation and Systems, vol. 5, no. 5, pp. 559-569, 2007.

[11] DexTAR, Parallel robot for education and research. Mecademic, 2014.

[12] T. D. Le, H.-J. Kang, and Y.-S. Suh, "Chattering-free neuro-sliding mode control of 2-dof planar parallel manipulators," International Journal of Advanced Robotic Systems, vol. 10, 2013.

[13] J.-P. Merlet, "Jacobian, manipulability, condition number, and accuracy of parallel robots," Journal of Mechanical Design, vol. 128, no. 1, pp. 199-206, 2006.

[14] H. Asada, "A geometrical representation of manipulator dynamics and its application to arm design," Journal of dynamic systems, measurement, and control, vol. 105, no. 3, pp. 131-142, 1983.

[15] B. Siciliano and O. Khatib, Springer handbook of robotics, ch. Performance Evaluation and Design Criteria. Springer Science \&amp; Business Media, 2008.

[16] F. Lara-Molina, K. Takano, and E. Koroishi, "Set-point regulation of a robot manipulator with flexible joints using fuzzy control," in 2015 12th Latin American Robotics Symposium and 2015 3rd Brazilian Symposium on Robotics (LARS-SBR), pp. 103-108, IEEE, 2015. 